\title{
Pneumonia and in-hospital mortality in the context of neurogenic oropharyngeal dysphagia (NOD) in stroke and a new NOD step-wise concept
}

\author{
G. W. Ickenstein · A. Riecker • C. Höhlig • \\ R. Müller · U. Becker $\cdot$ H. Reichmann • \\ M. Prosiegel
}

Received: 23 December 2009/Revised: 17 March 2010/Accepted: 19 March 2010/Published online: 10 April 2010

(C) The Author(s) 2010. This article is published with open access at Springerlink.com

\begin{abstract}
The aim of our work was to develop a step-wise concept for investigating neurogenic oropharyngeal dysphagia (NOD) that could be used by both trained nursing staff as well as swallowing therapists and physicians to identify patients with NOD at an early stage and so enable an appropriate therapy to be started. To achieve this objective, we assessed uniform terminology and standard operating procedures (SOP) in a new NOD step-wise concept. In-house stroke mortality rates and rates of pneumonia were measured over time (2003-2009) in order to show improvements in quality of care. In addition, outcome measures in a stroke-unit monitoring system were studied after neurorehabilitation (day 90) assessing quality of life (QL) and patient feedback. An investigation that was
\end{abstract}

G. W. Ickenstein $(\bowtie) \cdot C$. Höhlig

Department of Neurology and Stroke Unit, HELIOS General

Hospital Aue, Technical University Dresden, Gartenstr. 6, 08280 Aue, Germany

e-mail: guntram.ickenstein@helios-kliniken.de

A. Riecker

Department of Neurology, University Hospital Ulm,

Oberer Eselsberg 45, 89081 Ulm, Germany

R. Müller

Department of ENT, Carl Gustav Carus University Hospital,

Technical University Dresden, Fetscherstr. 74,

01307 Dresden, Germany

U. Becker · H. Reichmann

Department of Neurology, Carl Gustav Carus University

Hospital, Technical University Dresden, Fetscherstr. 40,

01307 Dresden, Germany

M. Prosiegel

Department of Neurology and Neurophysiology, m\&i Fachklinik Bad Heilbrunn, Wörnerweg 30, 83670 Bad Heilbrunn, Germany carried out in the context of internal and external quality assurance stroke projects revealed a significant correlation between the NOD step-wise concept and low rates of pneumonia and in-house mortality. The quality of life measures show a delta value that can contribute to "poststroke" depression. The NOD step-wise concept (NSC) should, on the one hand, be capable of being routinely used in clinical care and, on the other, being able to fulfil the requirements of being scientifically based for investigating different stages of swallowing disorders. The value of our NSC relates to the effective management of clinical resources and the provision of adequate diagnostic and therapeutic options for different grades of dysphagia. We anticipate that our concept will provide substantial support to physicians, as well as swallowing therapists, in clinical settings and rehabilitation facilities, thereby promoting better guidance and understanding of neurogenic dysphagia as a concept in acute and rehabilitation care, especially stroke-unit settings.

Keywords Neurogenic dysphagia - Acute stroke ·

Neurorehabilitation - Outcome research .

Screening for dysphagia

\section{Introduction}

Key quality data have been increasingly defined in hospitals on the basis of the diagnosis and procedure codes used for invoicing purposes, as well as demographics and quality control. In the HELIOS hospital system these data have been uniformly statistically recorded since the year 2002 [9]. Also, for outcome measurements, validated standard assessments are used in order to document the quality of life (QL) of the patients [21]. Despite the 
qualitative character of the construct "Quality of life (QL)" it is, for a number of reasons, meaningful to express QL parameters in terms of numerical values. A distinction is made between two measurement approaches depending on the type. On the one hand, there is the measurement approach extending across disorders, which is suitable for screening programs and which enables the investigator to make comparisons with other populations (healthy individuals, patients with other clinical pictures, patients from other countries, and cultures). On the other hand, there is the measuring approach specific to disorders which record the problems, needs, and experience of a clearly defined patient group and which is characterized by a high level of sensitivity. In this way, conclusions can be drawn about different therapy alternatives for patients with the same underlying affliction. The highest information content can be achieved by a combination of both approaches to measurement, but then a lower level of compliance on the part of patients must be expected. Not least, with the different approaches to measurement, the type of investigation is of importance. The various possibilities are outside assessments made by a physician (e.g., the Karnofsky Index, Spitzer test), and structured interviews with a standard form which has become most common in the meantime, e.g., questionnaires which are filled out by the patients themselves. Before a decision is made for this or that questionnaire or whether one's own questionnaire should be developed, it is always necessary to bring to mind the requirements demanded of a questionnaire designed to evaluate the quality of life [27].

The null hypothesis of this study was that with a new NOD step-wise concept (NSC) pneumonia and in-hospital mortality rates will not change over a period of 2 years (2007-2009) [13].

\section{Methods}

All stroke patients were evaluated with the NOD step-wise concept [11, 12]: first with a modified swallowing assessment (MSA) by the nursing staff on the day of admission, second followed with the clinical swallowing examination (CSE) by the swallowing therapist within the first $72 \mathrm{~h}$, and third with the flexible transnasal swallowing endoscopy (FTS) within 5 days by the physician. The study protocol was reviewed by the local ethical committee of the Technical University Dresden and has therefore been performed in accordance with the ethical standards laid down in the 1964 Declaration of Helsinki following GCP guidelines. All persons gave their informed consent prior to their inclusion in the study according to data safety standards (EK 246112006).
Modified swallowing assessment (MSA) in nursing care

Within the MSA questions need to be clarified whether the patient is awake, whether the patient can swallow his or her own saliva, whether the cough reflex is present, and whether he or she has fever attacks. Then the patient will be placed in an upright sitting position and requested to swallow, in order then to carry out an attempt to swallow a spoonful of non-carbonated water. In accordance with a protocol developed by Perry et al. [24] as well as Suiter and Leder [5, 32], we established a modified swallowing assessment (MSA) for the nursing staff (see Fig. 1) that assesses the following factors: dysarthria, dysphonia, a weakened impulse to cough, and an abnormal or absent pharyngeal reflex before the swallowing test, coughing and changes in the quality of the voice following the test. All items in the test must be answered with "no" before the 90-ml water test according to Suiter and Leder criteria can then be carried out to even detect mild to moderate signs of aspiration. Following the MSA, the nursing staff can record the abnormalities on a SOP sheet and notify the swallowing therapist and/or the doctor if signs of aspiration are detected. If appropriate, together they can lay down the first swallowing-adjusted diet (SAD). In danger of aspiration nothing per os (NPO) or feeding via a nasogastric tube (NGT) should be considered. In the event of mild to moderate swallowing difficulties a certain swallowingadjusted diet (with restrictions on consistency, soft-cooked or pureed food) can be recommended in advance. At the same time, nursing and kitchen personnel can uniformly establish a system using the NSC which certain types of food are suitable for the prescribed form of swallowingadjusted diet for each individual patient.

In-hospital mortality as a surrogate marker for the quality of medical care

Early rehabilitation is an essential component of the strokeunit concept. Within the context of neurological complex treatment, activating nursing care is carried out by nursing staff who have been trained in stroke-nurse courses in accordance with the standards of the German Stroke Society [15]. Swallowing therapists, speech, and language pathologists as well as ergotherapeutic and physiotherapeutic treatment concepts are implemented in the first days depending on the neurological deficits and are to be documented on a daily basis throughout the complex treatment. The particular methods such as Bobath, Taub's concept, proprioceptive neuromuscular facilitation (PNF), basal stimulation, cognitive training, and instruction of relatives are applied within the framework of a holistic concept. These complex tasks with corresponding early 


\section{Modified Swallowing Assessment (MSA)}

- Nursing staff/Swallowing therapist -

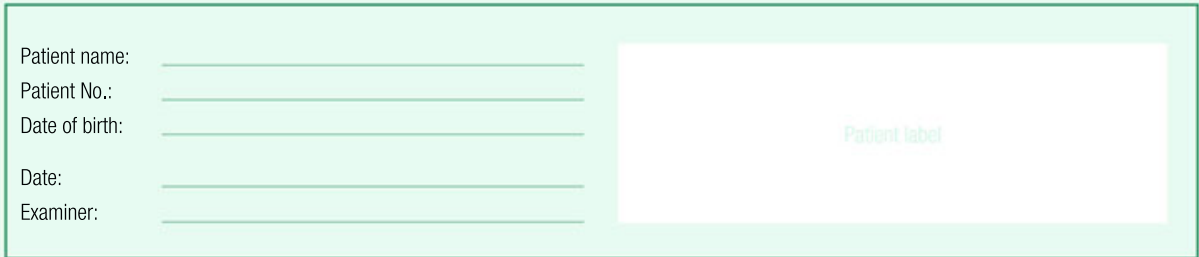

A - Swallowing Checklist (all patients)

\begin{tabular}{|c|c|c|c|}
\hline Suspicion of aspiration if answer ' $N$ o' & No & Yes & Comments \\
\hline a. Is the patient alert and responding to speech? & $\square$ & $\square$ & \\
\hline b. Can the patient cough when asked to? & $\square$ & $\square$ & \\
\hline c. Is the patient able to maintain some control of saliva? & $\square$ & $\square$ & \\
\hline d. Is the patient able to lick their top and bottom lip? & $\square$ & $\square$ & \\
\hline $\begin{array}{l}\text { e. Is the patient able to breathe freely (i. e. has no problem in breathing } \\
\text { without assistance and maintaining adequate oxygen saturation)? }\end{array}$ & $\square$ & $\square$ & \\
\hline f. Are signs of a wet- or hoarse-sounding voice absent? & $\square$ & $\square$ & \\
\hline
\end{tabular}

f. Are signs of a wet- or hoarse-sounding voice absent?

B - Swallowing test with 1 teaspoon of water (can only be performed if all points under " $\mathrm{A}$ " are "Yes")

- Patient sitting upright with trunk supported

- Mouth inspected for residues and asked to swallow saliva when prompted

- Palpation of swallowing, observe for symptoms when phonation prompted

\begin{tabular}{|c|c|c|c|}
\hline $\begin{array}{l}\text { Functional disturbance according to Perry criteria: } \\
\text { Terminate assessment if 'Yes' for any function }\end{array}$ & No & Yes & Comments \\
\hline a. No evident swallowing activity? & $\square$ & $\square$ & \\
\hline b. Water leaks out of the mouth? & $\square$ & $\square$ & \\
\hline c. Coughing/throat clearing? & $\square$ & $\square$ & \\
\hline d. Increase in respiratory rate? & $\square$ & $\square$ & \\
\hline e. Wet/gurgly voice within 1 minute immediately after swallowing? & $\square$ & $\square$ & \\
\hline f. Have you doubts or a bad impression? & $\square$ & $\square$ & \\
\hline
\end{tabular}

f. Have you doubts or a bad impression?

"No")

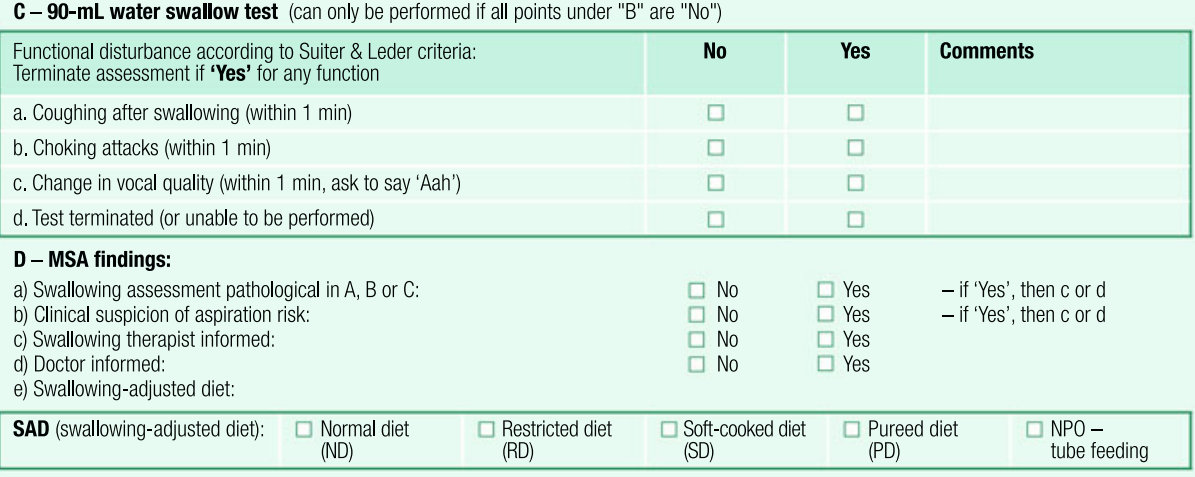

\begin{tabular}{lll} 
Comments: & \multicolumn{2}{l}{} \\
& Sate & Signature (Swallowing therapist) \\
\hline
\end{tabular}

Fig. 1 Modified swallowing assessment (MSA) for nursing staff (for more details concerning the NOD step-wise concept (NSC) please visit the website: http://www.helios-kliniken.de/klinik/aue/fachabteilungen/neurologie/neurogene-dysphagie.html)

diagnostics and therapy can only be guaranteed in a perfectly matched team that allows engagement of all its members. To achieve this aim, detailed consultation in the team is important in which doctors, stroke nurses, neuropsychologists, speech and language pathologists (SLP), ergotherapists, physiotherapists, as well as social workers are involved. For each patient, a special program of therapy (complex treatment and early rehabilitation) will be agreed on that will subsequently be continued in the geriatric or neuro-rehabilitation unit. In addition, difficult cases will be 
discussed in the stroke unit conference with all team members for internal further training [6].

An important, robust, and manipulation-safe outcome parameter is in-hospital mortality. However, there are numerous processes and intermediary quality result indicators available [3]. For internal purposes, we evaluate currently about 670 key data of which 142 are regularly observed as medical targets [1]. Target values are set on the basis of external comparison values (Federal Office of Statistics, 3M-DRG benchmarking sample, Federal Office of Quality Assurance (BQS), international literature etc.), provided that suitable data are available. For certain clinical pictures (e.g., cardiac heart failure, stroke), a risk adjustment is carried out according to age and gender. At the same time, a calculation is made of the expected mortality according to the hospital diagnosis data (Federal Office of Statistics) for a federal sample with the same risk structure and main diagnosis [30]. This is compared to the actual mortality found in the hospitals. The aim of every hospital is to achieve a mortality rate below the value expected on the basis of statistics [14]. Before and after the opening of the neurology department and the stroke unit in our hospital as well as the establishment of the NOD stepwise concept, an analysis of the stroke outcome was carried out in order to describe the improvements in the quality of care. In this analysis, the quality indicators were based on routine data and peer reviews. In addition, the medical care parameters were analyzed using the stroke-unit data sets and compared them to the data from the quality assurance project of northwest Germany.

Stroke-specific quality of life (SSQL) in dysphagic stroke patients as an outcome measure

The QL-questionnaire that we used "Quality of life of persons with swallowing complaints" was developed specifically in order to find out how swallowing disorders affect the quality of life. The QL assessment based on the SSQL (stroke-specific quality of life scale) [20] comprises, in total, 12 domains and 49 questions with a minimum of 49 and a maximum of 245 points to be scored with a cut-off point at 81 , meaning $<81$ points showing a poor outcome and $>81$ points showing a better outcome. However, it is important to know that a number of problems related to health may exist and it is sometimes difficult to unambiguously distinguish between these and swallowing complaints. A second questionnaire about "Satisfaction with the treatment of swallowing disorders" [27] was likewise used in order to find out how dysphagic patients view the quality of treatment for their swallowing disorder, including the corresponding consultation. It must be taken into account that, in our health care provision system, there are different therapeutical groups who concern themselves with swallowing disorders (e.g., speech and language pathologists, swallowing therapists, occupational therapists, trained nursing staff, etc.).

\section{Results}

Time measures of the NOD step-wise concept (NSC) were assessed in 114 stroke patients. For the MSA (modified swallowing assessment by nursing staff) $10.4 \mathrm{~min}( \pm 2.1)$ were needed on average by one of the stroke nurses. A clinical swallowing examination (CSE) by the swallowing therapist could be carried out comprehensively within $25.8 \mathrm{~min}( \pm 4.7)$. For the FTS (flexible transnasal swallowing endoscopy by the doctor/swallowing therapist) with testing of minimum two consistencies (pureed and fluid) about $34.3 \min ( \pm 6.3)$ were needed. The summarized NSC findings by the doctor/swallowing therapist documented within the computerized NSC program (http://www. eundl.de) required in total $6.8 \mathrm{~min}( \pm 1.8)$ so that, for the complete NSC-diagnostics, $76.5 \mathrm{~min}( \pm 4.9)$ on average was required.

Pneumonia rates, antibiotic consumption, and in-hospital mortality in the context of a stroke-unit monitoring system using the NOD step-wise concept

Within the context of the complex treatment in a strokeunit system, there is a clear necessity of carrying out clinical swallowing examinations (CSE), as well as more comprehensive equipment-based diagnostics using FTS or videofluoroscopic swallowing test (VFS) for a subsequent functional dysphagia therapy (FDT). The interaction of the various elements of quality management in a stroke unit were observed taking the in-hospital stroke mortality rate over the last six years (see Fig. 2).

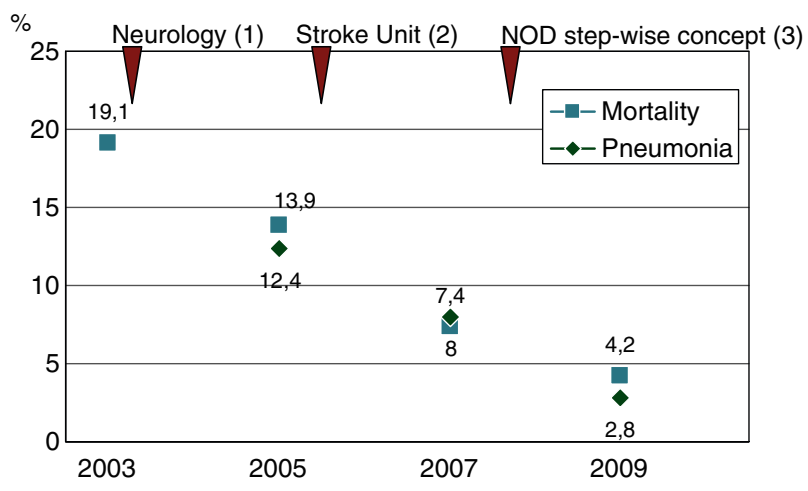

Fig. 2 In-hospital pneumonia and stroke mortality rate from 2003 to 2009 showing the relationship over the years between in-house stroke mortality rate $(\%)$ and the opening of the Department of Neurology (1), the opening of the Stroke Unit (2), and the introduction of an NOD step-wise concept (3) to reduce the rate of pneumonia and malnutrition with an structured dysphagia program 
Following the establishment of a neurology department in 2003 where all the stroke patients in the hospital were admitted, the mortality rate of our stroke patients declined from $19.1 \%$ (2003) to $13.9 \%$ (2005) and following the opening of the stroke unit to $7.4 \%$ (2007). After the NOD step-wise concept was introduced in 2007, the mortality rate was further reduced below the national average $(8.4 \%)$ to $4.2 \%$ (HELIOS quality assurance report 2009). Since the interregional certified stroke unit (registration number 1892886) is also part of the stroke quality assurance project 'northwest Germany', different key quality data for the treatment of stroke can be compared with the results of other stroke units in Germany or hospitals that treat stroke patients. With the help of the NOD step-wise concept, a structured dysphagia program was developed (MSA 100\%, CSE 66.1 vs. 54\%), which was reflected in a significantly reduced rate of pneumonia (2.8 vs. $9.0 \%$ average of all stroke units; $p<0.05$; odds ratio 0.26 , CI 0.09-0.71). Moreover, this reduced rate of pneumonia was also reflected in the consumption of antibiotics in our stroke unit. Compared to 2007, there was a significant savings by almost $50 \%$ in the cost of antibiotics (drug report 2009).

Specific quality of life (QL) on day 5 versus day 90

On day 5 and on day 90, the quality of life of the NOD patients was determined on the basis of the SSQL (strokespecific quality of life) scale, as well as the SWAL care questionnaire. In the group with a poor swallowing outcome and tube-feeding dependency (FCM 1-3 on day 90), $40.0 \%$ of patients ranked a low value for quality of life ( $<81$ points) on day 5 . In the group without feeding-tube dependency (FCM 4-7 on day 90) only 3.2\% had a score $<81$ on day 5 , i.e., indicating a better quality of life in this patient population $(p<0.000)$. With the continued existence of a clear swallowing disorder and feeding-tube dependence (FCM 1-3) on day 90, the renewed questioning on day 90 showed that now in the group with FCM 1-3 $55.1 \%$ of the patients had a value of $<81$, indicating a poor quality of life $(\Delta-\mathrm{QL}$ value $15.1 \%)$. In the group with a relatively good swallowing outcome on day 90 (FCM 4$7), 12.8 \%$ now reported a poor quality of life $(\Delta-\mathrm{QL}$ value $9.6 \%)$.

\section{Discussion}

Evaluation of the modified nursing assessment (MSA) in the context of the NOD step-wise concept

In clinical routine, a time delay between admission to the stroke unit and the commencement of dysphagia diagnostics is seen very frequently. During this interval, there is the possibility of aspiration as a result of food intake by the patient or, to some extent, also by the administration of food by relatives. Especially in the evening and at the weekends, at which time diagnostics and therapy are less frequent, there is often inadequate provision with regard to swallowing disorders. If there is an effective dysphagia program established with an initial swallowing assessment by the nursing staff or a clinical swallowing examination by a swallowing therapist or flexible trans-nasal swallowing endoscopy (FTS) by a physician, the risk of not recognizing a NOD is substantially reduced.

For this reason, a swallowing assessment by the nursing staff should be the first consideration for a $100 \%$ routine screening within a dysphagia program. In the annual stroke-nurse courses given by the German Stroke Society, this concept is intensively trained, in order to educate the nurses during half-day courses about neurogenic dysphagia and a modified swallowing assessment (MSA) [15], as well as the forms of diet to support swallowing and nutrition. During this initial testing with non-carbonated water, the risk of developing pneumonia with water aspiration can be considered to be very low [19, 35]. In one study, several water-swallow tests were carried out without a significant increase in aspiration pneumonia $72 \mathrm{~h}$ after testing [8]. However, the danger of aspiration pneumonia is disproportionately higher when, unaware of the presence of dysphagia, someone administers solid or purred food. With an initial modified protocol according to Perry et al. [24], which was especially developed for nursing staff, a sensitivity of 0.97 could be achieved.

Following the NOD step-wise concept that was designed to pass on consistent information, we established four NOD grades that can lead to special swallowing-adjusted diet (SAD) recommendations, so that an adequate form of nutrition can be found, passing on the information to the kitchen personnel. The NOD grade as a result of established scales (FCM = functional communication measure, PAS $=$ penetration-aspiration scale, $\quad$ SDS $=$ swallowing disability scale) $[22,23,26,29]$ is the sum of the swallowing disturbance pattern with following consequences and restrictions in diet: if there is no restriction at all, a NOD grade of 0 is given, i.e., the patient can be fed with a normal diet (SAD 0). When the patient has a mild dysphagia with a NOD grade of 1 , there is at least some disorder of bolus transport, so that restrictions and feeding under supervision is indicated (SAD 1). With a moderate NOD grade of 2, dietary restrictions will be necessary and fluids need to be thickened (SAD 2). With severe dysphagia and NOD grade 3 there is often a danger of penetration, so that nutrition should be given mostly via a feeding tube (SAD 3). In massive dysphagia with a NOD 
grade of 4 there is a danger of aspiration and therefore food should only be administered therapeutically to the patient by the swallowing therapist and a complete parenteral feeding via feeding tube (SAD 4) is necessary.

Neurogenic dysphagia in the context of complex treatment in the stroke-unit monitoring system

Bronchopneumonia is a frequent complication in the first few days following a brain infarct and is associated with a higher rate of mortality and an increased long-term impairment in those patients affected [28]. About 20\% of stroke patients who are admitted to a stroke unit suffer from early pneumonia following a stroke [10, 34]. Walter et al. showed that the presence of dysphagia alone is correlated with the highest relative risk of pneumonia. The study identified independent clinical predictors: the presence of dysphagia with a relative risk (RR) of 9.9, the existence of severe neurological deficits (NIHSS $\geq 10$ ) with an RR of 6.6 and signs of an infection (e.g., raised CRP on admission) with a RR of 3.8. Also, location of the brain infarct displayed an influence on the risk of pneumonia since it increased with the existence of a basal ganglia infarct (RR of 3.1). These findings could be confirmed in a follow-up study of dysphagic stroke patients and it was shown that an insular localization can be considered to be a predictor of early pneumonia [31]. A prospective study linked certain cerebral infarct localizations with specific, clinically and endoscopically characterized patterns of dysphagia. Thus, parietotemporal cerebral infarcts were associated with an attention deficit, left-sided parietotemporal cerebral infarcts with buccofacial apraxias, cerebral infarcts in the region of the upper motoneuron with orofacial paresis, and lateral medullary brainstem infarcts with an inadequate opening of the upper esophagus sphincter during swallowing.

The fact that in dysphagic stroke patients, who consequently already have an increased risk of pneumonia, an insular location of the brain infarct additionally increases the risk of infections, leads to an association of an additional disturbed neurohumoral immunogenic factor favoring infections [18]. Both a dysfunctional sympathetic nervous system as well as delayed regression of dysphagia are discussed. In addition, serum markers for adrenergic/ noradrenergic activation as well as an alteration in the immune system, such as an increase in interleukin 6 and a reduction in T-lymphocytes [2, 17, 33], have been postulated to be predictive of pneumonia. These findings are commensurate with animal studies that show a hyperactivation of the sympathetic system with consecutive immuno-suppression and early appearing of infections following a major cerebral infarct in the vascular territory of the middle cerebral artery (MCA) [25]. Questions remain as to whether a prophylactically administered betablocker or antibiotic therapy can improve the long-term outcome of stroke patients. A prerequisite for the use of prophylactic drug therapies is the definition of specific predictors of pneumonia, in order to select those groups of patients who can benefit from the early administration of prophylactic drugs.

\section{Quality of life of dysphagic stroke patients}

Quality of life (QL) is clearly influenced by the mode of taking food and the type and manner of preparation. The thickening of liquids, such as for example coffee or fruit juices, produces a clear loss in taste and is perceived by the patient as a drastic restriction in the quality of life. A similar experience is the reduction of oral food intake, for example resulting from the application of a feeding tube. In the measurement of the SLQL [27], it is postulated that with a score under 81 points the quality of life is experienced subjectively as "poor". The study result specifies the delta QL value, i.e., SLQL in the acute stage versus the SLQL after 90 days. In the acute stroke phase, a loss of QL was less frequently experienced than after 90 days, i.e., $\Delta 15 \%$. This could be interpreted that initially there is still great hope for therapy options with recovery from dysphagic stroke. Certainly, after 90 days, a more realistic estimation of the outcome prevailed and, in the case of patients with chronic dysphagia, results in a more pronounced reduction in quality of life. This subjectively experienced QL restriction could be most likely directed to "post-stroke depression", and it would be the task of a therapy based on anti-depressive drugs and psychotherapeutical support to overcome this negative effect on neurorehabilitation [7]. There are many citations in the literature that highlight the role of "post-stroke depression" as a reactive syndrome of restrictions on life in case of persisting stroke residuals [16]. At this point, it is important to consider the ethical aspects of feeding-tube applications and understand the meaning of QL. Artificial feeding should always be considered a form of medical treatment and this requires both a clear indication from the medical point of view as well as the authorization from the patient or his/her representative. Within this context, the situation can be seen quite differently in the case of a patient suffering from dementia, but in stroke patients, there often remains an unchanged feeling of hunger and thirst. A reduction of solid or liquid food should therefore be considered to be clearly unethical. For stroke patients, the application of a feeding tube is always part of the therapy, with the option of removing the tube when the "swallowing system" has recovered once again. The application of a feeding tube is not equated with the end of the swallowing therapy but, in fact, the basis for supplying 
the organism with energy in order to make collaboration in all forms of therapy possible.

\section{Conclusions}

Using our example of an early assessment of swallowing in a stroke-unit setting with the help of a NOD step-wise concept, the first results show that significant improvements in quality are possible, indeed to some extent producing clear reductions in the rate of pneumonia and costs for antibiotics. However, other factors that contribute to the results on rate of pneumonia, as well as mortality, must of course be taken into consideration [4]. Here, the increased sensitivity of all team members to these factors, the training of the specialized personnel (e.g., stroke nurses) in the area of neurogenic dysphagia, as well as in the area of nursing and therapeutical care should be mentioned. Through the implementation of a comprehensive stroke unit, a total process is encountered in which the NOD stepwise concept is only a small component of the complete chain of treatment. Nonetheless, these significant changes, which have been achieved and documented in a short period of time, cannot only by explained by the operating process. Rather, a clear effect of a dysphagia program can be recognized and should also be identifiable in other hospital settings to produce similar results. Since we have considered only a small part of stroke-unit patients, general recommendations can only be made with caution. The patient population selected was, however, well documented and analyzed in order to allow conclusions to be drawn for this group of dysphagic stroke patients, so that now further investigations based on our NOD step-wise concept can follow.

The complexity of neurogenic dysphagia and the great diversity of causes of the disorder require close cooperation between different medical and therapeutic specialists and disciplines with the establishment of special dysphagia laboratories. At the same time, continuous intensive training in the team must take place and structural steps have to be taken in order to design a dysphagia program. The data within the quality-assurance system, with low rates of pneumonia and a mortality rate lying well below the average for the country, confirm the efforts of all team members. Scales that deal with neurogenic dysphagia have, up until now, been considered to be the domain of rehabilitation centers, but what is important is commencement already in the acute stroke phase with an integrated transition to the rehabilitation phase.

Acknowledgments Bauer S, Buchheim M, Bräuer G, Dreischer T, Hofmayer A, Lindner-Pfleghar B, Müller A, Kerber K, Knoch S, Koch H, Kuch S, Pohl M, Pluschinski P, Schelling A, Schneider H,
Senf D, Sutarski S, Weber S, Wolf N. This work was supported by a grant from the HELIOS Research Center (HRC Berlin) and the expert group neuromedicine to G W Ickenstein (HEFÖ-ID 1481).

Open Access This article is distributed under the terms of the Creative Commons Attribution Noncommercial License which permits any noncommercial use, distribution, and reproduction in any medium, provided the original author(s) and source are credited.

\section{References}

1. AOK-Bundesverband, FEISA, HELIOS Kliniken, WIdO (Hrsg.) (2007) Qualitätssicherung der stationären Versorgung mit Routinedaten (QSR)-Abschlussbericht, Bonn

2. Chamorro A, Amaro S, Vargas M, Obach V, Cervera A, GómezChoco M, Torres F, Planas AM (2007) Catecholamines, infection, and death in acute ischemic stroke. J Neurol Sci 252(1):2935

3. Charlson ME, Pompei P, Ales KL, MacKenzie CR (1987) A new method of classifying prognostic comorbidity in longitudinal studies: development and validation. J Chronic Dis 40:373-383

4. Daniels SK, McAdam CP, Brailey K, Foundas AL (1997) Clinical assessment of swallowing and prediction of dysphagia severity. Am J Speech Lang Pathol 6:17-24

5. DePippo KL, Holas MA, Reding MJ (1992) Validation of the 3-oz water swallow test for aspiration following stroke. Arch Neurol 49(12):1259-1261

6. DSG, German Stroke Society (2009) Stroke units in Germany. Online Internet http://www.dsg-info.de

7. Finkenzeller W, Zobel I, Rietz S, Schramm E, Berger M (2009) Interpersonal psychotherapy and pharmacotherapy for post-stroke depression: feasibility and effectiveness. Nervenarzt 80:805-812

8. Garon BR, Engle M, Ormiston C (1997) A randomized control study to determine the effects of unlimited oral water intake in patients with identified aspiration. J Neuro Rehab 11:139-148

9. Heller G, Swart E, Mansky T (2003) Qualitätsanalysen mit Routinedaten. Ansatz und erste Analysen aus dem Gemeinschaftsprojekt „Qualitätssicherung mit Routinedaten“ (QSR) Klauber, Robra BP, Schellschmidt H (Hrsg.) Krankenhaus. J Report, pp 271-88

10. Hilker R, Poetter C, Findeisen N, Sobesky J, Jacobs A, Neveling M, Heiss WD (2003) Nosocomial pneumonia after acute stroke: implications for neurological intensive care medicine. Stroke 34(4):975-981

11. Ickenstein GW, Hofmayer A, Lindner-Pfleghar B, Pluschinski P, Riecker A, Schelling A, Prosiegel M (2009) Standardisierung des Untersuchungsablauf bei neurogener oropharyngealer Dysphagie (NOD). Neuro Rehabil 15(5):290-300

12. Ickenstein GW, Hofmayer A, Lindner-Pfleghar B, Pluschinski P, Riecker A, Schelling A, Prosiegel M (2009) Anhang zum NODStufenkonzept. Standardisierung der Diagnostik und Therapie bei neurogener oropharyngealer Dysphagie (NOD). Neuro Rehabil 15(6):342-354

13. Ickenstein GW, Stein J, Ambrosi D, Goldstein R, Horn M, Bogdahn U (2005) Predictors of survival after severe dysphagic stroke. J Neurol 252:1510-1516

14. Institute of medicine (2000) To err is human: building a safer health system. Institute of Medicine, Washington

15. Josten S (2009) What a stroke nurse does. Certified Nursing Education 1:20-21

16. Nurushima Kenji, Robinson RG (2007) Stroke related depression. Current Athero Report 4:296-303 
17. Klehmet J, Harms H, Richter M, Prass K, Volk HD, Dirnagl U, Meisel A, Meisel C (2009) Stroke induced immunodepression and poststroke infections: lessons from the preventive antibacterial therapy in stroke trial. Neuroscience 158(3):1184-1193

18. Koch HJ, Uyanik G, Bogdahn U, Ickenstein GW (2006) Relation between laterality and immune response after acute cerebral ischemia. Neuroimmunomodulation 13(1):8-12

19. Martino R, Silver F, Teasell R, Bayley M, Nicholson G, Streiner DL, Diamant NE (2009) The Toronto Bedside Swallowing Screening Test (TOR-BSST): development and validation of a dysphagia screening tool for patients with stroke. Stroke 40(2):555-561

20. McHorney CA, Robbins J, Lomax K, Rosenbek JC, Chignell K, Kramer AE, Bricker DE (2002) The SWAL-QOL and SWALCARE outcomes tool for oropharyngeal dysphagia in adults: III. Documentation of reliability and validity. Dysphagia 17:97-114

21. McMicken BL, Muzzy CL (2008) Functional outcomes of standard dysphagia treatment in first time documented stroke patients. Disabil Rehabil 26:1-12

22. Murray J, Langmore SE, Ginsberg S, Dostie A (1996) The significance of accumulated oropharyngeal secretions and swallowing frequency in predicting aspiration. Dysphagia 11:99-103

23. National Outcomes Measurement System (NOMS). ASHA 19982008, Rockville, Maryland

24. Perry L (2001) Screening swallowing function of patients with acute stroke. Part one: Identifications, implementation and initial evaluation of screening tool for use by nurses. J Clin Nurs 10(4):463-473

25. Prass K, Meisel C, Höflich C, Braun J, Halle E, Wolf T, Ruscher K, Victorov IV, Priller J, Dirnagl U, Volk HD, Meisel A (2003) Stroke-induced immunodeficiency promotes spontaneous bacterial infections and is mediated by sympathetic activation reversal by poststroke $\mathrm{T}$ helper cell type 1-like immunostimulation. J Exp Med 198(5):725-736
26. Prosiegel M, Heintze M, Wagner-Sonntag E, Hannig C, WuttgeHannig A, Yassouridis A (2002) Deglutition disorders in neurological patients. A prospective study of diagnosis, pattern of impairment, therapy and outcome. Nervenarzt 73:364-370

27. Prosiegel M, Wagner-Sonntag E, Koch F (2002) Fragebögen „Lebensqualität von Personen mit Schluckbeschwerden“ und „Zufriedenheit mit der Behandlung von Schluckstörungen“ "(2003) übersetzt aus der Originalarbeit von: McHorney and SWAL-CARE outcomes tool for oropharyngeal dysphagia in adults: III. Documentation of reliability and validity. Dysphagia 17:97-114

28. Ramsey D, Smithard D, Kalra L (2005) Silent aspiration: what do we know? Dysphagia 20:218-225

29. Rosenbek JC, Robbins JA, Roecker EB, Coyle JL, Wood JL (1996) A penetration-aspiration scale. Dysphagia 11:93-98

30. Schellschmidt H, Mansky T, Heller G, Robra BP (2007) Indikatoren und Messinstrumente zur Bewertung der medizinischen Ergebnisqualität im Krankenhaus. In: Stock J, Szecsenyi J (Hrsg.): Stichwort: Qualitätsindikatoren, Bonn

31. Steinhagen V, Grossmann A, Benecke R, Walter U (2009) Swallowing disturbance pattern relates to brain lesion location in acute stroke patients. Stroke 40(5):1903-1906

32. Suiter DM, Leder SB (2008) Clinical utility of the 3-ounce water swallow test. Dysphagia 23:244-250

33. Vogelgesang A, Grunwald U, Langner S, Jack R, Bröker BM, Kessler C, Dressel A (2008) Analysis of lymphocyte subsets in patients with stroke and their influence on infection after stroke. Stroke 39(1):237-241

34. Walter U, Knoblich R, Steinhagen V, Donat M, Benecke R, Kloth A (2007) Predictors of pneumonia in acute stroke patients admitted to a neurological intensive care unit. J Neurol 254(10):1323-1329

35. Zaidi NH, Smith HA, King SC et al (1995) Oxygen saturation on swallowing as a potential marker of aspiration in acute stroke. Age Ageing 24:267-270 